\title{
Tabularia
}

\section{Disparition ou conservation des sources et abandon de l'acte écrit : quelques observations sur les actes de Jumièges}

Disparition or conservation of the sources and interruption in the written practices: some observations on the charters of abbey of Jumièges

\section{Mathieu Arnoux}

\section{OpenEdition}

\section{Journals}

Édition électronique

URL : http://journals.openedition.org/tabularia/2010

DOI : 10.4000/tabularia. 2010

ISSN : $1630-7364$

Éditeur :

CRAHAM - Centre Michel de Boüard, Presses universitaires de Caen

Référence électronique

Mathieu Arnoux, «Disparition ou conservation des sources et abandon de l'acte écrit : quelques observations sur les actes de Jumièges », Tabularia [En ligne], Jumièges, foyer de production documentaire, mis en ligne le 22 octobre 2001, consulté le 21 avril 2019. URL : http:// journals.openedition.org/tabularia/2010; DOI : 10.4000/tabularia.2010 


\title{
Disparition ou conservation des sources et abandon de l'acte écrit : quelques observations sur les actes de Jumièges
}

\author{
Disparition or conservation of the sources \\ and interruption in the written practices: \\ some observations on the charters of abbey of Jumièges
}

\author{
Mathieu ARNOUX \\ Université Paris-7 Denis Diderot, EHESS \\ arnoux@ccr.jussieu.fr
}

Résumé:

La lacune documentaire des années 911-990 est l'un des principaux obstacles à la connaissance des origines du duché de Normandie. Il est nécessaire, pour donner tout leur sens aux rares sources subsistant pour cette période, de s'interroger sur la signification de cette interruption et les modalités d'un retour à l'utilisation de l'acte écrit à la fin du siècle. Le chartrier de Jumièges, où au moins un acte du $\mathrm{X}^{\mathrm{e}}$ siècle subsiste en original, constitue un bon observatoire pour l'usage des actes écrits aux $\mathrm{X}^{e}$ et XI $\mathrm{XI}^{\mathrm{e}}$ siècles. L'article s'attache en particulier à étudier le traitement réservé aux actes carolingiens de l'abbaye, qui furent copiés et utilisés en justice au XI ${ }^{\mathrm{e}}$ siècle. L'examen de ces actes suggère que, malgré l'arrêt de la production d'actes écrits dispositifs, les communautés religieuses du duché ne cessèrent pas de recopier et d'utiliser les monuments de leur histoire ancienne. Cette continuité de la conservation de l'écrit fut l'un des moyens de leur survie.

Mots-clés: Jumièges, IX'e-XI' siècles, Charles le Chauve, charte, diplôme, lacune documentaire.

\begin{abstract}
:
The documentary gap of the years 911-990 is one of the main obstacles to our knowledge of the origins of the Duchy of Normandy. It is necessary, to give their full meaning to the rare sources subsisting from that period, to question the signification of this interruption and the modalities of a return to the utilisation of written acts at the end of the century. The collection of charters of Jumièges, where at least one charter from the $10^{\text {th }} \mathrm{c}$. subsists in original form, represents a good observation point of the use of written charters in the $10^{\text {th }}$ and $11^{\text {th }}$ centuries. This paper is particularly engaged in the study of the treatment to which were subjected the Carolingian charters of the Abbey, which were copied and produced in court during the $11^{\text {th }}$ century. Examination of these deeds suggests that, in spite of the cessation of the production of written dispositive charters, the religious communities of the duchy never stopped copying and using the monumentae of their early history. This continuity in the preservation of written work was one of the means of their survival.
\end{abstract}

Keywords: Jumièges, $I X^{\text {th }}$-XI th century, Charles the Bald, charter, diploma, documentary gap. 
Il est entendu que la Normandie naît dans la seconde décennie du X $\mathrm{X}^{\mathrm{e}}$ siècle, lorsque le roi de France Charles le Simple cède au chef viking Rollon ses droits sur le comté de Rouen, et lorsque celui-ci et ses compagnons se convertissent au catholicisme et reçoivent le baptême des mains de l'archevêque Francon. Les sources sur l'événement, plus ou moins dignes de confiance, s'accordent sur la chronologie, la date de la cession des droits sur Rouen étant fixée à 911 par Dudon de SaintQuentin, celle du baptême à 912 par la Translation des reliques de saint Ouen, tandis qu'un diplôme de Charles le Simple pour l'abbaye Saint-Germain-des-Prés en 918 évoque la récente installation à Rouen des Normands de Rollon ${ }^{1}$. Ce très célèbre document, conservé en original, est à la fois la première source sûre et l'ultime témoignage direct sur l'histoire précoce de la Normandie ${ }^{2}$. Il faudra attendre la charte de fondation de la collégiale de Fécamp, rédigée en 990, pour trouver un acte normand relatif au duché de Normandie. Autour de Richard I Ir apparaissent alors une hiérarchie ecclésiastique au grand complet et un groupe laïc complexe, où se devinent déjà certains des traits de l'entourage ducal au siècle suivant. Dans l'intervalle sont nés un pouvoir nouveau et une société nouvelle. La genèse de l'État ducal s'accomplit donc dans une obscurité qui s'étend sur la quasi-totalité du Xe siècle.

Il y a là un défi que doit relever tout historien de la société normande. Il est indispensable de discerner, au-delà de cette discontinuité documentaire, ce qui peut être discontinuité historique et ce qui, au contraire, peut être pensé par induction à partir des sources antérieures, ou des sources voisines ${ }^{3}$. On pourrait objecter que l'absence des sources invite l'historien prudent à la retenue, ou au silence. C'est là se dérober à l'obligation de rendre compte de l'écoulement du temps, et de la continuité de la durée historique: se taire serait accréditer par défaut l'hypothèse qu'il ne s'est rien passé dans la province ecclésiastique de Rouen entre 918 et 990, alors que tout suggère qu'il s'agit d'une période fondamentale, non seulement pour l'histoire interne du duché en formation, mais aussi pour l'équilibre instable d'un Regnum Francorum en voie de recomposition. Les deux questions liées auxquelles doit répondre l'historien sont donc les suivantes: que s'est-il passé dans les terres normandes au $\mathrm{X}^{\mathrm{e}}$ siècle ? Et comment arriver à le savoir? On s'intéressera ici à la deuxième de ces interrogations.

L'absence de sources diplomatiques entre 918 et 990 requiert description et explication: s'il s'agit d'une complète disparition de sources ayant existé, l'historien est condamné à en prendre son parti. Si au contraire il s'agit d'une interruption de l'usage de l'écrit suivie de sa reprise, il y a là un fait social positif, qui se prête à l'interprétation. L'ensemble des sources sur la province de Rouen et sur la Normandie

1. GulLLot, Olivier, «La conversion des Normands peu après 911 », Cahiers de civilisation médiévale, XXIV, 1981, p. 101-116 et 181-219.

2. LAUER, Philippe, Recueil des actes de Charles III le Simple, roi de France (893-923), t. 1, Paris, Académie des Inscriptions et Belles-Lettres (Chartes et diplômes relatifs à l'histoire de France), 1940, p. 209-212, nº 92 .

3. Cf. sur le cas analogue de la Gascogne les remarques de B. Cursente dans Les sociétés méridionales autour de l'an Mil: répertoire des sources et documents commentés, Michel ZimmermanN (éd.), Paris, CNRS, 1992, p. 259-260. 
ducale est bien connu pour la période qui nous intéresse : il y a peu à ajouter à la liste des actes du haut Moyen Âge dressée en 1946 par Lucien Musset ${ }^{4}$, au recueil des actes ducaux de Marie Fauroux, ou à l'inventaire des sources de la Normandie du $\mathrm{XI}^{\mathrm{e}}$ siècle, récemment proposé par Emily Tabuteau ${ }^{5}$. Il suffit de les associer pour que le vide documentaire du $\mathrm{X}^{\mathrm{e}}$ siècle apparaisse dans son ampleur : sur 108 actes antérieurs à l'an Mil du catalogue de L. Musset, 12 seulement peuvent être rapportés à la période 900-1000; 7 d'entre eux concernent des localités extérieures au duché. La conclusion est la même pour les actes ducaux: des 6 actes antérieurs à 1000 où figure la souscription ducale, 2 sont extérieurs au duché, 2 sont incertains ou faux, 2 seulement paraissent authentiques ${ }^{6}$. Il est d'ailleurs plus simple de recenser les quelques sources fiables parvenues jusqu'à nous. La liste est courte: une confirmation des biens de l'abbaye de Saint-Évroult par Charles le Simple $(900)^{7}$, un diplôme du même roi à son chancelier Ernustus, relatif à des domaines de la région rouennaise $(905)^{8}$, et le diplôme déjà cité de 918 remettant à l'abbaye de Saint-Germain-des-Prés la tutelle sur l'abbaye de La Croix-Saint-Ouen, sont tout ce qui nous reste pour le début du siècle. Pour les décennies suivantes, on ne conserve que trois actes relatifs aux affaires normandes, émanant d'autorités extérieures au duché. Les deux plus anciens sont, sinon faux, du moins fort remaniés: il s'agit d'une bulle de Jean XIII accordant la liberté d'élection abbatiale aux moines récemment installés au Mont-Saint-Michel et d'un diplôme du roi Lothaire de 966 confirmant la fondation monastique et les clauses de la bulle pontificale? . Seule paraît insoupçonnable la notice d'un plaid tenu à Gisors en

4. MUSSET, Lucien, « Notes pour servir d'introduction à l'histoire foncière de la Normandie : les domaines de l'époque franque et les destinées du régime domanial du IX ${ }^{\mathrm{c}}$ au XI siècle », Bulletin de la Société des antiquaires de Normandie, t. 49, 1946, p. 7-97, aux p. 80-92. Signalons en complément des actes cités la mention en 833 d'une possession de Marmoutier dans l'Avranchin: éd. DE TRÉMAULT, Auguste, Cartulaire vendômois de Marmoutier, Vendôme, 1893, $\mathrm{n}^{\circ} 1$ (donation du comte Troannus, texte signalé par Dominique BARThÉlemy, La société dans le comté de Vendôme de l'an Mil au XVe siècle, Paris, Fayard, 1993, p. 452).

5. ZACK-TABUTEAU, Emily, Transfers of Property in eleventh century Norman Law, Chapell Hill (University of North-Carolina Press), 1988, p. 250-268.

6. Fauroux, Marie, Recueil des actes des ducs de Normandie (911-1066), Caen (MSAN, t. XXXVI), 1961 : les $n^{\text {os }} 1$ (faux) et 2 , sont extérieurs au duché; le $n^{\circ}$ 6/28, perdu, pourrait aussi bien être de Richard II et le $\mathrm{n}^{\circ} 5$ est sûrement faux (WERNER, Karl Ferdinand, «Quelques observations au sujet des débuts du "duché” de Normandie», Droit privé et institutions régionales. Études historiques offertes à J. Yver, Paris, publications de l'université de Rouen, 1976, p. 691-709, à la p. 692, n. 4), du moins dans la version qui nous en est parvenue (notons cependant la fondation très ancienne de l'abbaye Saint-Taurin : son abbé assiste, peut-être avant 990, à la translation des reliques de saint Ouen [P. L. 162, col. 1162] ; il est donc tout à fait possible qu'il y ait eu une charte de Richard I ${ }^{\text {er }}$ pour Saint-Taurin; de ce qui en subsiste dans l'acte qui nous est parvenu, on ne voit pas qu'elle ait eu l'apparence monumentale du diplôme fécampois); en fin de compte, des actes publiés par M. Fauroux, seuls les n ${ }^{\text {os }} 3$ et 4 échappent à la critique.

7. LAUER, Philippe, Recueil des actes de Charles III le Simple..., p. 74, $\mathrm{n}^{\circ} 35$.

8. Ibidem, p. $112, \mathrm{n}^{\circ} 51$.

9. Parmi les nombreuses éditions qui ont été données de l'un et de l'autre acte, et dans l'attente de l'édition du cartulaire préparée par K. Keats-Rohan, il convient de préférer celle de la chronique du cartulaire du Mont-Saint-Michel éditée par E. de Robillard de Beaurepaire dans LE RoY, Thomas, Les Curieuses recherches du Mont-Saint-Michel, Caen, Mémoires de la Société des antiquaires de 
968 par Hugues Capet et Richard Ir ${ }^{\text {er }}$ pour confirmer aux moines de Saint-Denis la possession du domaine de Berneval, près de Dieppe. Il s'agit de textes à la fois isolés et marginaux, pris sur les frontières de l'État normand. Le premier diplôme émis par un souverain normand pour un établissement normand paraît bien être la charte de fondation de la Trinité de Fécamp, le 15 juin $990^{10}$. Comme prévu, la récolte est maigre: aucun acte écrit en territoire normand entre 911 et 990 n'a survécu jusqu'à nous. Ajoutons pour faire bonne mesure que des quelque vingt mentions de donations ducales antérieures à l'an Mil recueillies par Marie Fauroux dans les actes subsistants, aucune ne fait allusion à un acte écrit ${ }^{11}$. Rien ne permet donc de démontrer que des actes écrits aient été produits en Normandie avant 990 . Il reste à décider si cette absence est le résultat de la disparition de toutes les archives ${ }^{12}$ ou d'une cessation de l'activité d'écriture.

Il est évidemment possible que ce soit la conséquence des destructions advenues au cours des IX ${ }^{\mathrm{e}}$ et $\mathrm{X}^{\mathrm{e}}$ siècles. Rien ne permet pourtant d'être affirmatif sur ce

Normandie, 1878, p. 872-873), texte de propagande où le lien et la fonction des deux actes apparaissent parfaitement. La bulle de Jean XIII est depuis longtemps reconnue comme un faux grossier; comme le fait remarquer H. Guillotel (CHÉDEVILLE, André, et GuILLOTEL, Hubert, La Bretagne des saints et des rois. $\mathrm{V}^{\mathrm{e}}-\mathrm{X}^{\mathrm{e}}$ siècle, Rennes, Ouest-France, 1984, p. 336), il n'y a aucune raison de réserver un traitement de faveur au diplôme de Lothaire (HALPHEN, Louis et LOT, Ferdinand, Recueil des actes de Lothaire et de Louis V, Paris, Académie des Inscriptions et Belles-Lettres (Chartes et diplômes relatifs à l'histoire de France), 1908, n 24, p. 53-57), qui ne vaut pas mieux; qu'il ait existé au $\mathrm{X}^{\mathrm{e}}$ siècle une bulle de Jean XIII et un diplôme de Lothaire pour le Mont-Saint-Michel est par ailleurs possible, mais indémontrable à partir de ces actes.

10. FAUrouX, Marie, Recueil..., n ${ }^{\circ} 4$, p. 72-74. Un autre texte aujourd'hui disparu dispute au diplôme fécampois son antériorité, dont il faut écarter les prétentions : il s'agit de la charte de fondation de la collégiale de La Ferté-en-Bray, que dom Toussaint Duplessis vit dans le chartrier de Saint-Laurenten-Lyons (Description géographique et historique de la haute Normandie, Paris, 1740, t. 1, p. 116-117, t. 2, p. 322). Il l'attribue à Richard $I^{\text {er }}$ et le date de 989 ou 990 ; les éléments de datation qu'il donne pour cet acte fort important renvoient au règne de Richard II, qui souscrit l'acte en compagnie de son fils le futur Richard III et de son frère Robert, archevêque de Rouen, ainsi que de Robert comte de Mortain, et d'un évêque Hugues, qui pourrait être titulaire du siège de Coutances, de Bayeux ou de Sées; sûrement authentique, l'acte est donc à dater des années 1015-1026.

11. Fauroux, Marie, Recueil..., p. 19-24.

12. La plus grande partie de celles-ci n'est même pas soupçonnable; le polyptyque d'Irminon, qui nous a conservé la description du domaine de Villa-supra-Mare (il peut s'agir de Saint-Germain-Village [Eure, ar. Bernay, c. Pont-Audemer], comme le propose Dietrich LOHRMANN ( «e moulin à eau dans le cadre de l'économie rurale de la Neustrie (VII--IX ${ }^{\mathrm{e}}$ siècles) ", in La Neustrie. Les pays au nord de la Loire de 650 à 850, Colloque historique international publié par Hartmut Atsma, Beihefte der Francia, 16/1 et 2, Sigmaringen, 1989, p. 367-404), suivi sur ce point par Dieter HäGERMANN, Das Polyptychon von Saint-Germain-des-Prés. Studienausgabe, Cologne, éd. Bohlau, 1993, p. 228 ou bien de Villers-sur-Mer [Calvados, ar. Lisieux, c. Trouville], selon la recherche récente de Christophe Maneuvrier, Paysages et sociétés rurales au Moyen Âge. Le pays d'Auge jusqu'à la fin du XIII' siècle, thèse de l'Université de Caen, 2000, dactyl., p. 72), nous livre cependant une allusion fugitive à des biens situés dans le bocage virois, à Tallevende (Breve xxiv, de Bisconcella, 10: Isigilus, colonus Sancti Germani, de Talevinda, et uxor extranea: A. LONGNON, Polyptique de Saint-Germain des prés rédigé au temps de l'abbé Irminon, Paris, 1895, repr. Genève, 1978, t. 2, p. 320 ; HÄGERMAN, éd. cit. p. 192); correctement identifié par Auguste Longnon à Saint-Germain de Tallevende (Calvados, ar. et c. Vire), venus à l'époque ducale à l'abbaye de Saint-Sever, Talevinda est identifié par le dernier éditeur comme un nom de personne (HÄGERMAN, éd. cit., index, p. 258). 
point. Malgré les pertes considérables subies par les communautés religieuses et l'émigration hors de la province de la plupart d'entre elles, plusieurs ont conservé jusqu'au XI ${ }^{e}$ siècle des éléments de leurs chartriers anciens, preuve que la destruction ne fut pas totale. La chose est évidente dans le cas de la cathédrale de Rouen et de l'abbaye Saint-Ouen, dont les fonds renferment encore deux actes carolingiens originaux, à preuve d'une continuité effective dans la conservation des actes antérieurs. D'autres actes prénormands nous sont parvenus pour les abbayes de Jumièges et Saint-Wandrille dans des copies plus tardives, le plus souvent du XI ${ }^{\mathrm{e}}$ siècle. Le statut diplomatique de ces actes, sans doute devenus en partie inintelligibles à l'époque ducale, n'est pas clair et a sans doute influé sur leur conservation. Il est un indice de l'usage ou de l'abandon de l'écriture aux premiers temps du duché.

La communauté la plus richement dotée en actes et sources du haut Moyen Âge était Saint-Wandrille; les notices des Gesta Sanctorum patrum Fontanellensis Coenobii, ainsi que les Vitae de ses fondateurs, rédigées à l'époque carolingienne et précieusement conservées, y racontaient l'histoire de la communauté et de son temporel. Tous ces textes furent recopiés au XI' siècle dans le Chronicon maius de l'abbaye ${ }^{13}$. À côté de ces textes historiques et hagiographiques, qui servirent aux $\mathrm{XI}^{\mathrm{e}}$ et $\mathrm{XII}^{\mathrm{e}}$ siècles à forger des faux diplômes de Thierry III et Childebert III ${ }^{14}$, quelques chartes anciennes avaient survécu en original. Un diplôme d'immunité concédé par Louis le Pieux en 815 fut transcrit, en copie figurée, à la fin du XI siècle dans le Chronicon maius. Le même diplôme fut, semble-t-il, recopié au début du XIV siècle dans une partie aujourd'hui disparue du grand cartulaire de l'abbaye ${ }^{15}$. La plupart de ces actes anciens firent l'objet d'un traitement particulier, qui révèle la valeur qui leur était attribuée; notons cependant qu'une charte de 882 fut transcrite au cartulaire au début du XIV sans qu'aucun signe ou commentaire marque son originalité parmi les quelques 2700 actes conservés dans les feuillets du manuscrit ${ }^{16}$.

\section{Jumièges face à son passé}

Le cas de Jumièges est différent : bien que ses actes carolingiens ne soient conservés en original (mais elle possède une confirmation délivrée en 984 par l'évêque de Chartres pour ses possessions de Vaux, dans le Vexin ${ }^{17}$ ), sa bibliothèque et son chartrier constituent un observatoire remarquable sur l'usage qu'une communauté pouvait faire des monuments de son histoire. Il est vrai que l'histoire de la communauté, si

13. Lot, Ferdinand, Études critiques sur l'abbaye de Saint-Wandrille, Paris (Bibliothèque de l'École des hautes études, $n^{\circ}$ 204), 1913; dom LOHIER, Fernand et dom LAPORTE, Jean, Gesta sanctorum patrum Fontanellensis coenobii, Rouen (Société d'Histoire de la Normandie), Paris, 1936.

14. LOT, Ferdinand, Études critiques..., p. 23-26, nº 1 et 2.

15. Ibidem, p. 28-30, nº 4 ; p. 29, n. 1.

16. Ibidem, p. $36-37, \mathrm{n}^{\circ} 6$.

17. Cf. l'édition, traduction et commentaire de ce texte par N. Manoury dans La Normandie vers l'an Mil, François de Beaurepaire et Jean-Pierre Chaline (éd.), Rouen (Société de l'histoire de la Normandie), 2000, p. 116-119. 
difficile à reconstituer pour la période qui suit l'abandon de son site originel après 866, est au centre de l'histoire des origines du duché. C'est sûrement dans l'entourage de l'abbé Martin, restaurateur de l'abbaye vers 942, que fut composé le Planctus pour la mort de Guillaume Longue Épée, le plus ancien texte littéraire original composé dans le duché ${ }^{18}$. Sa bibliothèque, sans doute la plus riche de la province de Rouen, constitue aussi une voie d'accès privilégiée pour son étude: elle comprend en particulier plusieurs manuscrits hagiographiques remontant au $\mathrm{X}^{\mathrm{e}}$ siècle, qui témoignent de la volonté de retrouver les sources neustriennes de l'histoire de la Normandie ${ }^{19}$. L'abondance dans ses manuscrits de textes hagiographiques d'origine irlandaise, récemment mise en lumière par J.-M. Picard, témoigne peut-être aussi de la même fidélité à son histoire la plus ancienne ${ }^{20}$. Dans d'autres cas, les religieux semblent avoir considéré ces actes comme des preuves parmi d'autres de leurs droits, et les utilisèrent comme tels.

La tradition des actes carolingiens de Jumièges ouvre d'autres perspectives sur la fonction et l'usage des monuments de la communauté. Les textes de deux diplômes nous ont été conservés, l'un de Pépin I ${ }^{\text {er }}$ d'Aquitaine, de 838, notifiant la restitution à l'abbaye de son domaine de Tourtenay, l'autre de Charles le Chauve, confirmant en 849 la division des biens de l'abbaye entre la mense abbatiale et la mense conventuelle, et énumérant les possessions de cette dernière ${ }^{21}$. Ces deux diplômes nous sont parvenus dans des copies figurées du XIe siècle, dont l'existence, et encore plus la qualité témoignent de l'intérêt apporté alors à ces monuments de l'histoire de la communauté. Un examen paléographique et diplomatique très minutieux permettrait peut-être de pré-

18. BECKER, Phillipp August, «Der Planctus auf den Normannenherzog Wilhelm Langschwert (942) », Zeitschrift für Franzözische Sprache und Literatur, 63, p. 190-197; les références à l'abbé Martin constituent un indice sérieux sur la provenance du texte. L'un des deux manuscrits de l'œuvre, actuellement à la Bibliothèque laurentienne de Florence (ms Libri Ashburnam 83) provient apparemment de la bibliothèque d'Orléans; il n'est pas impossible qu'il s'agisse à l'origine d'un manuscrit de Saint-Mesmin de Micy, dont Annon, successeur de Martin à Jumièges, fut aussi l'abbé (cf. note suivante). Cf. aussi LEMARIGNIER, Jean-François, «Jumièges et le monachisme occidental au Haut Moyen Âge (VII $-\mathrm{XI}^{\mathrm{e}}$ s.) », Jumièges. Congrès scientifique du XIII centenaire, Rouen, Lecerf, 1956, p. 753-764.

19. La présence dans la bibliothèque de cette abbaye d'un manuscrit hagiographique du $\mathrm{X}^{\mathrm{e}}$ siècle (Rouen, Bibl. mun., ms 1378/U 40) écrit sur ordre de l’abbé Annon, devenu après 943 abbé de Saint-Mesmin de Micy incite à mettre en doute la disparition effective de la communauté de Jumièges après l'assassinat de Guillaume Longue Épée en 943. Il semble bien que l'abbaye ait conservé certains manuscrits apportés de Poitiers par l'abbé Martin vers 942, ce qui constitue un argument en faveur de sa continuité en Normandie.

20. PICARD, Jean-Michel, «Les saints irlandais en Normandie», Les Saints dans la Normandie médiévale, Pierre Bouet et François Neveux (éd.), Caen, PUC, 2000, p. 49-69.

21. LeVILlain, Léon, Recueil des actes de Pépin Ier et de Pépin II, rois d'Aquitaine (814-848), Paris, Académie des Inscriptions et Belles-Lettres (Chartes et diplômes relatifs à l'histoire de France, 7), 1926, $\mathrm{n}^{\circ} 29$, p. 124-126 et VERNIER, Jules-J., Chartes de l'abbaye de Jumièges (v. 825-1204), Rouen-Paris (Société de l'Histoire de Normandie), 1916, t. 1, p. 2-5, nº 2 ; Tessier, Georges, Recueil des actes de Charles II le Chauve, roi de France, vol. 1, Paris Académie des Inscriptions et Belles-Lettres (Chartes et diplômes relatifs à l'histoire de France, 9), 1943, n 111, p. 293-297 et VERNIER, Jules-J., Chartes de l'abbaye de Jumièges..., t. 1, p. 5-11, $\mathrm{n}^{\circ} 3$. 
ciser la date de ces copies et leurs rapports. On serait tenté de penser que l'acte de Pépin fut recopié au moment où Jumièges conclut avec l'abbaye de Bourgueil l'échange des domaines de Tourtenay et de Longueville, en 1012. La copie B du diplôme de Charles le Chauve, que Georges Tessier date de la fin du X $\mathrm{X}^{\mathrm{e}}$ siècle ou du début du XIe siècle, fut peut-être faite à la même occasion, le diplôme faisant lui aussi mention du domaine de Tourtenay. Par la suite, le domaine de Tourtenay ayant cessé d'appartenir à la communauté, le diplôme de Pépin, toujours conservé dans le chartrier, ne fut plus recopié.

Celui de Charles le Chauve, au contraire, continua à servir de preuve, et connut par la suite une carrière diplomatique et juridique notable. La copie $\mathrm{C}$ du texte en est le témoignage. Quasiment identique à la copie B, elle n'en diffère, pour le texte du diplôme, que par l'ajout au début de la date d'une formule comprenant millésime, épacte, indiction, concurrent et cycle pascal, tout à fait incompatible avec la datation par l'année du règne présente sur l'original. L'analyse du document ne permet pas de décider si cette interpolation, différente dans son écriture du reste de la ligne, fut portée dans une copie sincère établie antérieurement, ou si elle demanda l'établissement d'une nouvelle copie. À la suite du diplôme, a été recopiée, d'une main de la fin du XI ${ }^{e}$ siècle, une brève notice relative à l'investiture du domaine de Curwahan, concédé à Gosselin de Coulonces par un abbé de Jumièges non autrement désigné. La copie porte au dos, d'une écriture du XI ${ }^{\mathrm{e}}$ siècle, la mention Curtwaham, aujourd'hui Courgains, dans la Sarthe. Le même texte se retrouve à la suite de la notice originale d'un plaid tenu par Robert de Bellême en 1086, pour juger de la restitution aux moines de Jumièges de la terre voisine de Dame-Marie, qui leur avait été donnée en 1023-1027 par Albert, abbé de SaintMesmin de Micy, et dont ils avaient perdu la jouissance ${ }^{22}$. Bien qu'aucun lien ne soit établi entre les deux notices, il est vraisemblable que la seconde rapporte une décision prise au cours du même plaid où siégèrent aux côtés de l'abbé de Jumièges ceux de Saint-Martin de Sées et de Saint-Pierre-de-la-Couture du Mans. Une copie du diplôme de Charles le Chauve, pourvue pour l'occasion d'un millésime faux, mais sûrement de bon aloi pour les juges, fut alors exhibée à la cour; pour des raisons archivistiques, la décision fut par la suite inscrite au bas du texte ${ }^{23}$. Selon les termes même de la notice, il ne paraît pas que la démarche ait eu le moindre succès : l'abbaye de Saint-Vincent du Mans avait reçu des biens à Courgains dès le pontificat d'Avesgaud, évêque du Mans (995-1032) ${ }^{24}$. L'affaire ne se termina

22. Arch. dép. Seine-Maritime, 9H 1433; VerNIER, Jules-J., Chartes de l'abbaye de Jumièges..., t. 1, p. 110$113, \mathrm{n}^{\circ} 34$; MUSSET, Lucien, «Administration et justice dans une grande baronnie normande au $\mathrm{XI}^{\mathrm{e}}$ siècle : les terres des Bellême sous Roger II et Robert », MUSSET, Lucien, BouVRIS, Jean-Michel, MAILlEFER, Jean-Marie, Autour du pouvoir ducal normand. Xe-XIIe siècles, Caen (Cahier des Annales de Normandie, $\left.\mathrm{n}^{\circ} 17\right), 1985$, p. 129-148, à la p. 134.

23. Le diplôme de Charles le Chauve entra dans une liasse Curvaham, tandis que la notice de plaid rejoignait la donation de l'abbé Albert dans la liasse Dame-Marie (9H 1433).

24. LOUISE, Gérard, La seigneurie de Bellême, X ${ }^{\mathrm{e}-X I I}{ }^{\mathrm{e}}$ siècles, Le Pays bas-normand, 199-202, Flers, 19901991, t. 1, p. 325-236; GuILlOT, Olivier, Le comte d'Anjou et son entourage au XIe siècle, Paris, 1972, t. 2 , p. $178, \mathrm{n}^{\circ} 268$ (1041-1068). 
pas là : à deux reprises dans le demi-siècle qui suivit, les moines de Jumièges contestèrent aux moines manceaux la possession de l'église de Courgains. Ils le firent sans trop de conviction: une notice mancelle de 1129 raconte que les religieux, pourtant présents au Mans, renoncèrent à se présenter au plaid tenu dans la curia episcopi sur la propriété des églises de Courgains et de Saosnes ${ }^{25}$. Lorsque le débat fut rouvert en 1148, ils produisirent à nouveau au juge l'acte (instrumentum) de Charles le Chauve. Sans contester son ancienneté, leurs adversaires firent valoir contre elle l'absence de sceau, d'autant qu'ils produisaient eux-mêmes un acte scellé de l'évêque Hildebert de Lavardin ${ }^{26}$. La conclusion qu'on peut tirer de cette série de procédures n'est pas parfaitement probante: même munis d'un titre authentique, les moines de Jumièges ne parvinrent pas à faire valoir leur bon droit. Elle montre cependant qu'un diplôme de ce genre pouvait être allégué en justice à preuve de ses prétentions : la conservation des actes les plus anciens trouvait ainsi sa justification.

Antérieurement au plaid de 1086, l'histoire normande avait connu des cas d'usage des textes prénormands à des fins de restitution. Les moines de l'abbaye Saint-Florent de Saumur s'adressèrent ainsi en 1055-1056, pour récupérer les terres qui leur avaient autrefois été données dans le Cotentin, et dont ils donnaient la liste, sans doute tirée de sources d'époque mérovingienne. La donation par Guillaume le Conquérant de Flottemanville-Hague, dans le nord du Cotentin, répond apparemment à la requête des religieux. Des toponymes énumérés dans leur lettre, seul un avait pu être identifié et avait donc été restitué ${ }^{27}$. C'est une revendication du même genre qui, dans la première moitié du XIe siècle, conduisit les moines de Saint-Wandrille à forger, à partir d'extraits du Chronicon maius, un faux diplôme de Thierry III relatif à l'île de Belcinnaca qui avait été donnée par ledit roi à saint Condède ${ }^{28}$. Le document dut impressionner car on en retrouve des passages entiers dans la charte donnée par Guillaume d'Arques à l'occasion de la restitution du domaine en question. Peut-être les moines de Jumièges eurent-ils recours en 1027 à un écrit de même nature pour obtenir de Robert le Pieux la restitution d'une terre proche de leur domaine de Montataire, quam ex sancte Bathilde tempore Gemmeticenses tenuerant monachi ${ }^{29}$. Mais le plus bel exemple d'utilisation d'un diplôme ancien nous est offert par Robert, archevêque de Rouen, qui, vers 1035, fit ajouter les souscriptions du duc Robert le Libéral et de Guillaume, fils de celui-ci, au bas d'un diplôme de Charles le Chauve, ainsi

25. Vernier, Jules-J., Chartes de l'abbaye de Jumièges..., t. 1, p. 151-154.

26. Liber controversiarum Sancti Vincentii Cenomannensis, André CHÉdEVILLE (éd.), Paris, 1968, n 267 ; cf. Bruno LemesLE, La société aristocratique dans le Haut-Maine (XI ${ }^{e}$-XII ${ }^{e}$ siècles), Rennes, PUR, 1999, p. 50 et 62-63.

27. MARChegay, Paul, «Chartes normandes de l'abbaye de Saint-Florent près Saumur de 710 à 1200 », Mémoires de la Société des antiquaires de Normandie, t. 30, 1880, p. 668-672; FAUROUX, Marie, Recueil..., p. 386-387, n 199.

28. Lot, Ferdinand, Études critiques..., p. 23-24, n ${ }^{\circ} 1$ et p. 56-57, n 15 (1032-1047).

29. Vernier, Jules-J., Chartes de l'abbaye de Jumièges..., t. 1, p. 27-29, n 11. 
que sur les diplômes qui récapitulaient l'évolution du temporel de la métropole dans les premiers temps du duché ${ }^{30}$.

La transmission et l'usage des actes diplomatiques prénormands suggèrent donc qu'un certain nombre d'établissements religieux d'origine neustrienne ne perdirent jamais véritablement leur familiarité avec les monuments anciens de leur histoire ${ }^{31}$. L'extension de l'enquête au-delà des sources diplomatiques montrerait que l'usage de l'écrit existe dans le duché dès que les institutions religieuses sont en place. La chose est certaine pour l'archevêché de Rouen, qui resta sur place en permanence. Diverses sources attestent que cette continuité de l'institution se traduisit dans la tenue des archives: seul de tous les évêchés normands, le siège rouennais dispose de listes épiscopales précises et complètes pour le $\mathrm{X}^{\mathrm{e}}$ siècle. Il serait possible de regrouper autour de cette source officielle d'autres textes, comme ceux reçus par l'archevêque Gui de son confrère Hervé, archevêque de Reims, auquel il avait écrit au sujet des problèmes posés par la conversion des Normands établis à Rouen. Ou comme, pour la fin du siècle, les poésies satiriques de Garnier de Rouen, qui témoignent de l'existence d'écoles autour du cloître de la métropole ${ }^{32}$. Débordant des frontières du duché, l'archevêché devait d'ailleurs s'adapter aux règles en usage dans le royaume de France pour la gestion de ses droits dans l'archidiaconé de Vexin. Une charte de 979-989 concédée par l'archevêque Hugues aux moines de Saint-Germain des Prés en donne l'illustration: c'est, pour autant qu'on puisse le savoir, le plus ancien acte émis par une institution interne au duché ${ }^{33}$.

Il y a donc bien eu, malgré l'ampleur des destructions de toutes sortes, volonté de transmettre à la postérité les traces écrites de l'histoire ancienne des communautés religieuses. Cela vaut aussi bien pour les communautés émigrées hors de la province de Rouen durant une partie du $\mathrm{X}^{\mathrm{e}}$ siècle, comme Jumièges ou Saint-Wandrille, que pour Saint-Ouen de Rouen, réinstallé dès avant 920, ou l'archevêché, qui resta sans interruption dans la ville métropolitaine : ces deux dernières institutions sont les seules à avoir conservé des originaux, comme si, toutes proportions gardées, l'exil avait été plus néfaste aux archives que la permanence sur place.

Le sens et l'autorité attachés à ces textes semblent avoir varié. Seuls le hasard de la conservation et le scrupule des copistes justifient l'inscription dans le grand cartulaire de Saint-Wandrille de la donation par Nithard en 882 d'un courtil de 30

30. FAuroux, Marie, Recueil..., n ${ }^{\circ} 68$ et 66 et 67.

31. L'exemple de l'abbaye de Saint-Evroult (supra n. 30) suggère, a contrario, que la récupération tardive de ce type de document ne permet pas forcément d'enrichir la mémoire de la communauté.

32. Musset, Lucien, «Le satiriste Garnier de Rouen et son milieu (début du XI ${ }^{\mathrm{e}}$ siècle) », Revue du Moyen Âge latin, t. 10, 1954, p. 237-266.

33. GUILlot, Olivier, «La conversion des Normands... »; POUPARDIN, René, Recueil des chartes de l'abbaye de Saint-Germain-des-Prés des origines au début du XIII siècle, Paris, Société de l'Histoire de Paris et de l'Ile-de-France, 1909, p. 73-74, n 44; la souscription Robertus archiepiscopus venant à la suite de celle de l'archevêque Hugues, ne fait pas problème: elle s'explique par la correction d'un copiste scrupuleux, veillant à compléter la suscription de Robert, fils du duc Richard, après qu'il fut parvenu au siège épiscopal (l'acte ne nous est connu que par une copie du XII' siècle dans le Cartulaire +++ Arch. nat. LL 1024, fol. 60). 
perches situé à Ramcharias, dans un pagus dont le scribe n'a pas réussi à lire le nom ${ }^{34}$, ce qui prive l'acte de toute utilité, et la conservation par les moines de Jumièges d'un acte du IX ${ }^{\mathrm{e}}$ siècle relatif à un domaine qu'ils avaient depuis longtemps cédé. Les moines de Saint-Evroult semblent pour leur part avoir été embarrassés par le diplôme de 900 de Charles le Simple confirmant les biens de leur abbaye, que leur abbé Robert de Grandmesnil rapporta d'Orléans où il était allé demander la restitution des reliques de saint Évremond: ils ne le firent pas figurer dans leur cartulaire et Orderic Vital, qui copia de sa main la version qui nous en est parvenue, n'en fit même pas mention dans son œuvre ${ }^{35}$. Cet acte délivré à une communauté de chanoines ne pouvait peut-être pas être d'une grande utilité aux bénédictins installés dans l'abbaye, qui accordèrent beaucoup plus d'importance au retour des corps de leurs fondateurs qu'à la redécouverte de leur histoire.

De cette brève revue des sources écrites normandes avant 990 ressort confirmée l'impression générale d'une dramatique régression de l'écrit dans le duché au $\mathrm{X}^{\mathrm{e}}$ siècle; il n'est pas possible cependant d'affirmer qu'il y ait totale destruction de toute culture écrite: dès qu'il y a continuité des institutions religieuses, des sources apparaissent, marginales, extérieures au duché le plus souvent, mais qui témoignent que la compétence d'écriture ne s'est jamais complètement perdue, en particulier dans la région rouennaise. Dès lors, on doit penser qu'il y a une part de choix dans l'abandon de l'écriture, et plus encore dans le retour à un enregistrement écrit des actes dans les dernières années du règne de Richard $\mathrm{I}^{\mathrm{er}}$. Sa participation à des actes émis par d'autres souverains peut donc être vue comme une préparation au couronnement de son règne, la restauration de l'abbaye de Fécamp, moment essentiel de la stabilisation et du développement de l'État ducal.

34. LOT, Ferdinand, Études critiques..., p. 36-37, n 6; voir aussi H. Van Werveke, «Saint-Wandrille et Saint-Pierre de Gand (IX ${ }^{\mathrm{e}}$ et $\mathrm{X}^{\mathrm{e}}$ siècles) », Miscellanea medievalia in memoriam Jan Frederik Niermeyer, Groningen, 1967, p. 79-92.

35. Orderic Vital, Histoire ecclésiastique, éd. M. Chibnall, t. 3, p. XVI-XVII et 323-324. 